\title{
Primary Hepatic Leiomyosarcoma: a Case Report and Review of the Literature
}

\author{
Themistoklis Feretis ${ }^{1}$, loannis D. Kostakis ${ }^{1}$, Christos Damaskos ${ }^{1, *}$, Nikolaos Garmpis ${ }^{1}$, Dimitrios Mantas ${ }^{1}$, \\ Afroditi Nonni ${ }^{2}$, Gregory Kouraklis ${ }^{1}$, Dimitrios Dimitroulis ${ }^{1}$
}

\begin{abstract}
Background/Aim: Primary hepatic leiomyosarcoma is an extremely rare type of liver sarcoma with relatively poor prognosis, with about 50 cases having been reported in the literature. Potential origins of this tumor in the liver are the smooth muscle cells in the round ligament, intrahepatic blood vessels and intrahepatic bile ducts. There is no apparent sex predilection and there is a wide age range. The clinical presentation is not specific and the diagnosis depends on the expression of markers such as smooth muscle actin, desmin and vimentin by tumor cells.

Patients and Methods: Herein, we present a case of a bulky primary hepatic leiomyosarcoma in a 68-year-old female patient. The patient underwent resection of the exophytic mass en block with the hepatic segments III and IVB.

Conclusion: Surgical resection is the most effective among the treatment options.

\section{KEYWORDS}

hepatic; liver; leiomyosarcoma, primary

AUTHOR AFFILIATIONS

${ }^{1}$ Second Department of Propedeutic Surgery, "Laiko" General Hospital, National and Kapodistrian University of Athens, Medical School, Athens, Greece

2 First Department of Pathology, National and Kapodistrian University of Athens, Medical School, Athens, Greece

* Second Department of Propedeutic Surgery, Laiko General Hospital, National and Kapodistrian University of Athens, Medical School; 17 Agiou Thoma Street, Athens, 11527, Greece; e-mail: x_damaskos@yahoo.gr
\end{abstract}

Received: 26 May 2018

Accepted: 5 November 2018

Published online: 22 January 2019

Acta Medica (Hradec Králové) 2018; 61(4): 153-157

https://doi.org/10.14712/18059694.2018.135

(c) 2018 The Authors. This is an open-access article distributed under the terms of the Creative Commons Attribution License (http://creativecommons.org/licenses/by/4.0), which permits unrestricted use, distribution, and reproduction in any medium, provided the original author and source are credited. 


\section{INTRODUCTION}

Primary hepatic sarcomas are rare tumors including various types, such as angiosarcoma, fibrosarcoma, liposarcoma, embryonal sarcoma, malignant fibrous histiocytoma, carcinosarcoma and epithelioid hemangioendothelioma (1). Primary hepatic leiomyosarcoma is another extremely rare type of liver sarcoma with relatively poor prognosis, with about 50 cases having been reported in the literature. Herein, we report a case of a bulky primary hepatic leiomyosarcoma in a 68-year-old female patient.

\section{CASE REPORT}

A 68-year-old female patient was referred to our department in order to treat a bulky liver tumor. The patient has been complaining for right upper quadrant heaviness and discomfort for 1 year. She underwent an abdominal ultrasound scan which revealed the presence of a hepatic mass located in the left lobe of the liver. Subsequently, she underwent an abdominal and thoracic computed tomography and an abdominal magnetic resonance tomography that confirmed the presence of a bulky, exophytic and heterogeneously attenuated mass

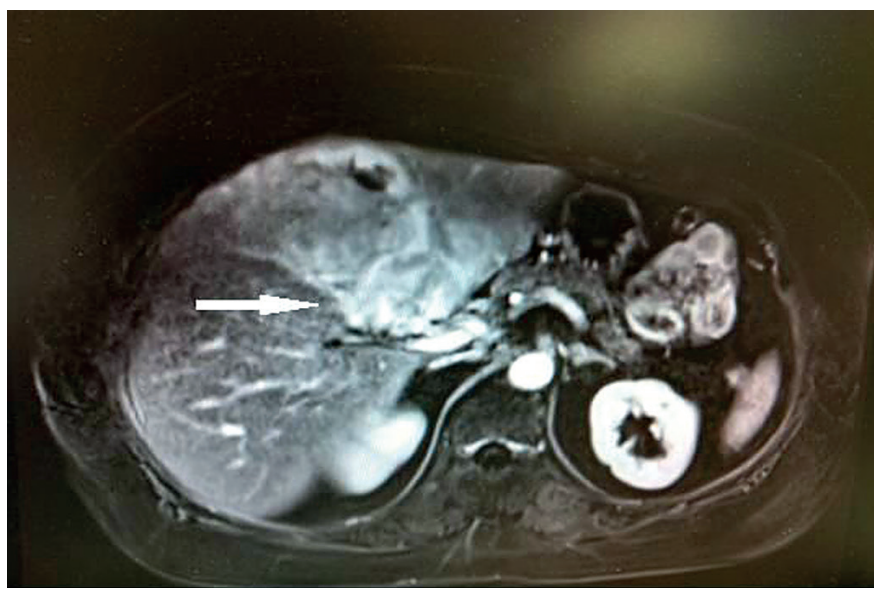

Fig. 1 Abdominal magnetic resonance imaging. The white arrow shows the liver tumor.

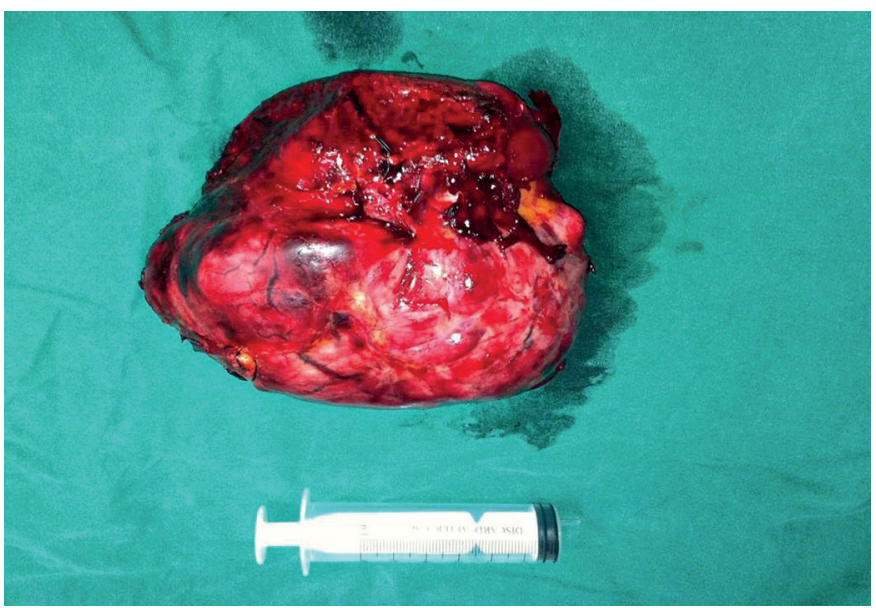

Fig. 2 Surgical specimen. with irregular margins, originating from the hepatic segments III and IVB (Figure 1). The tumor was $13 \mathrm{~cm}$ in diameter. The imaging studies excluded the presence of other primary sites. The patient had a medical history of chronic hepatitis B, type 2 diabetes mellitus and cholelithiasis. The patient's complete blood count test, biochemical tests and levels of carcinoembryonic antigen, alpha fetoprotein and cancer antigen 19-9 were within normal range.

The patient underwent resection of the exophytic mass en block with the hepatic segments III and IVB, from which the tumor appeared to be originated, along with cholecystectomy (Figure 2). The postoperative course was uneventful and the patient was discharged on the 7th postoperative day. The histopathological examination showed the presence of a whitish tumor, $13 \mathrm{~cm}$ in diameter, with central cystic degeneration. The tumor was extended up to Glisson's capsule and up to $2 \mathrm{~mm}$ from the resection margins. The neoplasm was a sarcoma made of large, atypical cells with abundant cytoplasm that occasionally were multinucleated (Figure $3 \mathrm{~A}$ ). The tumor included regions of spindle-shaped cells with fascicular arrangement and foci of osteoid production (Figure 3B). Extensive regions of necrosis were also present. No intracellular globules or entrapped bile ducts were detected. The neoplasm encased small blood vessels without infiltrating them. The mitotic count was 10 mitoses per 10 high-power fields (HPF). Furthermore, the immunohistochemical examination of the sarcoma revealed extensive expression of smooth muscle actin (Figure 3C) and limited expression of desmin (Figure 3D), but did not detect any expression of myoD1, myogenin, S-100 protein, c-kit or cytokeratines 7, 8, 18, 19 and 20, excluding diagnoses such as rhabdomyosarcoma, melanoma, gastrointestinal stromal tumor (GIST), carcinoma, etc. Therefore, a diagnosis of primary pleomorphic hepatic leiomyosarcoma was established.

The patient received six cycles of chemotherapy postoperatively with cyclophosphamide, doxorubicin and vincristine. Due to the presence of advanced tumor, she also received pazopanib, a multi-targeted receptor tyrosine kinase inhibitor, per os, which has been added as a treatment option for the

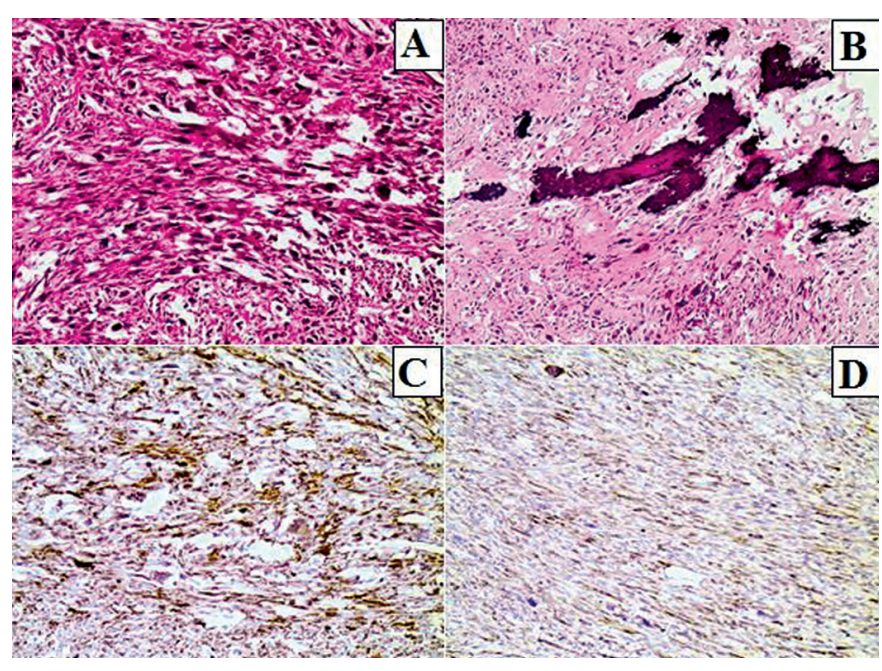

Fig. 3 Histological images. (A) Sarcoma made of large, atypical cells with abundant cytoplasm (hematoxylin-eosin stain). (B) Foci of osteoid production (hematoxylin-eosin stain). (C) Extensive expression of smooth muscle actin. (D) Limited expression of desmin. 


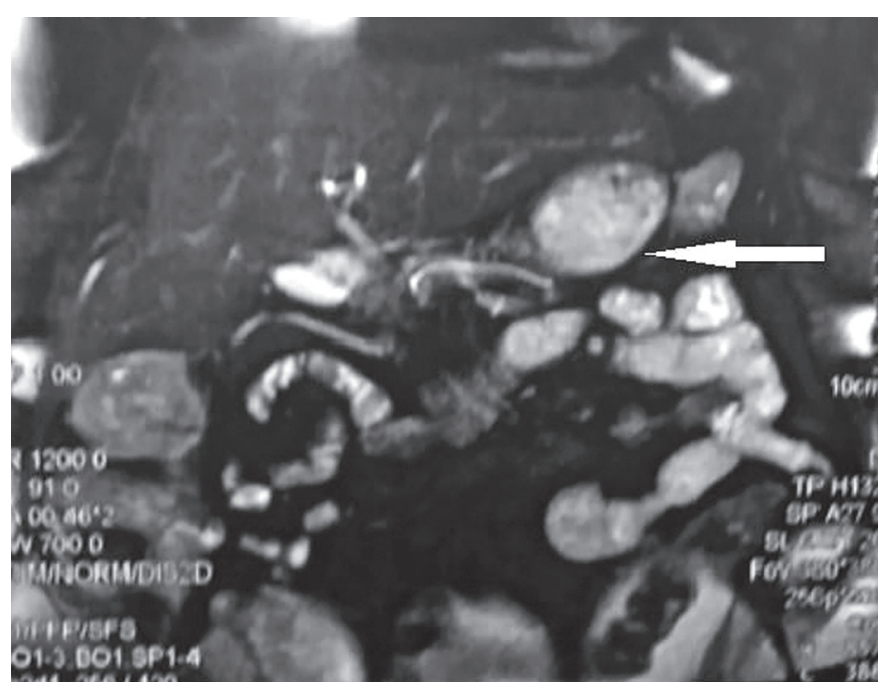

Fig. 4 Abdominal magnetic resonance imaging. The white arrow shows one site of recurrence.

advanced, metastatic and/or unresectable sarcomas, apart from liposarcomas (2, 3). An abdominal magnetic resonance tomography 18 months after the operation revealed four sites of recurrence, one between the liver and the first portion of duodenum, $5.5 \mathrm{~cm}$ in diameter, other two at the front of pylorus, $3 \mathrm{~cm}$ and $2.2 \mathrm{~cm}$ in diameter, and another one at the front of the body of stomach, $2.1 \mathrm{~cm}$ in diameter (Figure 4). The recurrent tumors were removed surgically, the postoperative course was uneventful and the patient was discharged from the hospital on the 7th postoperative day. She continued the per os treatment with pazopanib.

However, 3 months after the second operation, the patient presented melenas. An upper gastrointestinal endoscopy showed infiltration of the anterior wall of the first portion of duodenum by an ulcerated neoplasm. An abdominal computed tomography revealed three new sites of recurrence: one between the liver, the portal vein and the second portion of duodenum; another in contact with the anterior wall of the stomach and the first portion of duodenum, $5.5 \mathrm{~cm}$ in diameter; and another arising from the mesocolon and reaching the abdominal wall (Figure 5). The patient received conservative treatment and continued the per os treatment with pazopanib. There was gradual progression of the disease and the patient died 37 months after the initial diagnosis from acute upper gastrointestinal bleeding due to infiltration of the duodenum by the tumor.

\section{DISCUSSION}

Primary hepatic leiomyosarcoma is an extremely rare malignant disease, with 50 cases having been reported in the literature so far, our case included (4-40). It is a malignant tumor that arises from smooth muscle cells $(4,8,10-13,15,17,19$, $21,22,26,28,29,31-33,36-38,40)$. Potential origins of this tumor in the liver are the smooth muscle cells in the round ligament $(29,31,37,38)$, the intrahepatic blood vessels and the intrahepatic bile ducts $(15,17,22,26,29,31,32,37,38$, 40). There is no apparent sex predilection, since there have been reported $23(46 \%)$ cases of male patients $(5,9,10,12$, $13,15,17,19,20,24-26,28-30,32-35,37)$ and $27(54 \%)$ cases

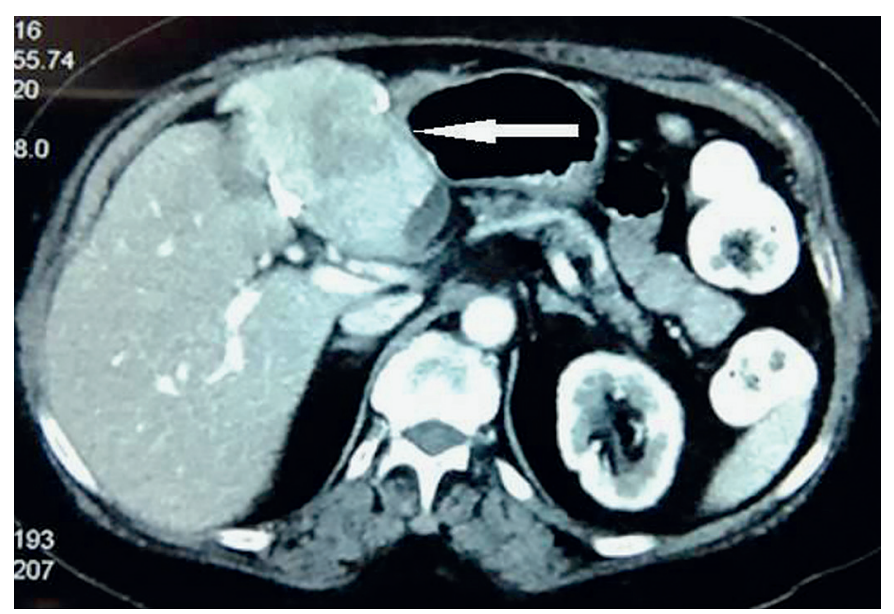

Fig. 5 Abdominal computed tomography. The white arrow shows one site of new recurrence.

of female patients, including our patient $(4,6-8,11,13,14$, $16-18,21-23,25,27,28,31,36,38-40)$. The mean patients' age is 51.3 years and the median patients' age is 58 years. However, there is a wide age range, since the youngest patient was 5 months old (31) and the oldest patient was 86 years old (11), and the standard deviation is 18.6 years.

The underlying pathogenetic mechanisms have not been identified yet. However, there are some cases of primary hepatic leiomyosarcoma in immunosuppressed patients, two of whom had acquired immunodeficiency syndrome (32, 33). One of the patients with acquired immunodeficiency syndrome also had infection from Epstein-Barr virus (32). Another patient with primary hepatic leiomyosarcoma was under immunosuppressive treatment after renal transplantation (21). Furthermore, there are two cases of primary hepatic leiomyosarcoma in patients with chronic hepatitis, one with hepatitis C (19) and the other is our patient, who had hepatitis B.

Concerning the clinical image of primary hepatic leiomyosarcomas, they are often asymptomatic $(21,25,30)$ or their symptoms are non-specific $(4,8,10-12,14,16,17,19,21,23$, $27-29,31,32,34,37,38,40)$. Abdominal pain or discomfort $(4,8,11,12,14,16,17,19,22,23,27-29,34,37,38)$, a palpable mass $(4,19,22,28,29,38)$, fever $(17,23,28,31,32,40)$, jaundice (39), anorexia $(10,12,27,28,32)$, nausea or vomiting $(31,34)$ and weight loss $(4,8,10,12,14,16,19,28,32,37)$ are among the reported manifestations. A case with acute bleeding has also been reported (24). There is usually a single mass $(4,8,11-13,16,18-25,27-36,38-40)$, although there are cases with two $(25,37)$, three $(26)$ or even multiple tumors $(15)$. The size of the tumor at diagnosis varies greatly $(4,8,11,12$, $15,16,18-23,25-35,37-40)$ with the smallest tumor being $0.6 \mathrm{~cm}$ in diameter (35) and the largest one being $30 \mathrm{~cm}$ in diameter (11). The mean diameter of all the reported primary hepatic leiomyosarcomas is $10.3 \mathrm{~cm}$ and the median diameter is $9.1 \mathrm{~cm}$, whereas the standard deviation is $6.7 \mathrm{~cm}$. The distribution of these tumors within the liver differs between the right and left hemiliver, with two thirds of them having risen from the right hemiliver $(4,8,11,13,16,17,19-22,25-28,31$, $32,34,35)$ and one third from the left hemiliver $(12,18,25,26$, $29,30,33,36-40)$. 
There is a number of data about radiological and histological findings of primary hepatic leiomyosarcoma in the literature. Ultrasonography usually shows a hypoechoic mass $(15,17,19,29,30,33,37)$ or a mass with heterogeneous echogenicity $(17,22,27,30,31,37)$. Computed tomography usually reveals a hypodense $(12,17,19,21,23,27-33,37,39)$ and often heterogeneous mass $(17,21,23,31,34)$ with inhomogeneous and often peripheral enhancement after administration of intravenous contrast $(15,17,19,23,27-33,40)$, which may show regions of cystic degeneration (27, 28, 31, 32). Magnetic resonance imaging displays on the other hand a usually heterogeneous mass that is hypointense in T1-weighted images and hyperintense in T2-weighted images (16, 19, 21, 37, 39, 40). Histological examination of primary hepatic leiomyosarcomas shows spindle-shaped cells with fascicular arrangement $(4,8,10-12,15-17,19,21,22,26-34,36-38,40)$. Immunohistochemistry reveals expression of smooth muscle actin (13, $15,18,19,21,22,27-33,36-38,40)$, desmin $(13,15,19,21,22$, $27-34,36-38)$ and vimentin $(15,17,19,22,27-29,31,33,37$, $38,40)$, whereas cytokeratins $(22,27-33,37,38)$, neuron-specific enolase $(27-29,32)$ and S-100 protein $(19,21,27-34,37$, $38,40)$ are not expressed.

Regarding therapeutic options for primary hepatic leiomyosarcoma, hepatic resection, in the form of wedge resection, segmentectomy, lobectomy or extended hepatectomy, was the preferred method for tumors without distant metastases $(4,6-8,11,12,14,16,17,20-23,25,26,28-31,33-36$, 38). However, there were four patients with tumors confined within the liver who underwent liver transplantation $(25,27$, $39)$. Some authors reported the addition of adjuvant chemotherapy consisted of various combinations of drugs $(6,8,10$, $23,28,30,31,35,37,39)$. Some of the administrated chemotherapeutic regimens were the following: 1 . vincristine, cyclophosphamide and dactinomycin (8); 2. cyclophosphamide, vincristine and actinomycin D (10); 3. doxorubicin (23); 4. folinic acid, fluorouracil, irinotecan (FOLFIRI) and bevacizumab (30); 5. mitoxantrone, cisplatin and fluorouracil (35); 6. ifosfamide and mesna (37). Radiotherapy has also been used as an adjuvant treatment along with chemotherapy in three cases $(10,23,28)$. Finally, transarterial chemoembolization has also been used in one case (35) and transarterial infusion of epirubicin and carboplatin in another case (37). Survival rates are relatively low. On the basis of articles reporting survival data, median survival is 37.5 months and 5 -year survival rate is $40 \%$ (4-12, 14, 17, 20-23, 25-38, 40).

In conclusion, primary hepatic leiomyosarcoma is a rare malignant disease with relatively poor prognosis. The most preferred type of treatment is surgical excision, which sometimes is combined with adjuvant chemotherapy and/or radiotherapy. However, very little is known about the effectiveness of the current treatment because of the rarity of the disease. More in-depth studies are needed to investigate and shed light on this uncommon clinical entity.

\section{CONFLICTS OF INTEREST}

The authors declare that they have no conflicts of interest.

\section{REFERENCES}

1. Yu RS, Chen Y, Jiang B, et al. Primary hepatic sarcomas: CT findings. Eur Radiol. 2008; 18: 2196-205.

2. von Mehren $M$, Randall RL, Benjamin RS, et al. NCCN clinical practice guidelines in oncology. Soft tissue sarcoma. Version 2.2018. 2018; $1-49$.

3. Casali PG, Abecassis N, Bauer S, et al. Soft tissue and visceral sarcomas: ESMO-EURACAN. Clinical Practice Guidelines for diagnosis, treatment and follow-up. Ann Oncol 2018; 29(Suppl. 4): iv51-iv67.

4. Wilson SE, Braitman H, Plested WG, et al. Primary leiomyosarcoma of the liver. Ann Surg 1971; 174: 232-7.

5. Fong JA, Ruebner BH. Primary leiomyosarcoma of the liver. Hum Pathol 1974; 5: 115-9.

6. Masur H, Sussman EB, Molander DW. Primary hepatic leiomyosarcoma: A report of two cases. Gastroenterology 1975; 69: 994-7.

7. Yoshikawa K, Satake K, Kinoshita H, et al. Primary leiomyosarcoma of the liver. Clin Oncol 1977; 3: 197-202.

8. Bloustein PA. Hepatic leiomyosarcoma: ultrastructural study and review of the differential diagnosis. Hum Pathol 1978; 9: 713-5.

9. O'Leary MR, Hill RB, Levine RA. Peritoneoscopic diagnosis of primary leiomyosarcoma of liver. Hum Pathol 1982; 13: 76-8.

10. Chen KT. Hepatic leiomyosarcoma. J Surg Oncol 1983; 24: 325-8.

11. Maki HS, Hubert BC, Sajjad SM, et al. Primary hepatic leiomyosarcoma. Arch Surg 1987; 122: 1193-6.

12. Paraskevopoulos JA, Stephenson TJ, Dennison AR. Primary leiomyosarcoma of the liver. HPB Surg 1991; 4: 157-62.

13. Watanabe K, Saito A, Wakabayashi H, et al. Two autopsy cases of primary leiomyosarcoma of the liver. Superiority of muscle-specific actin immunoreactivity in diagnosis. Acta Pathol Jpn 1991; 41: 461-5.

14. Baur M1, Pötzi R, Lochs $\mathrm{H}$, et al. Primary leiomyosarcoma of the liver-a case report. Z Gastroenterol 1993; 31: 20-3.

15. Holloway H, Walsh CB, Thomas R, et al. Primary hepatic leiomyosarcoma. J Clin Gastroenterol 1996; 23: 131-3.

16. Soyer P, Blanc F, Vissuzaine $C$, et al. Primary leiomyosarcoma of the liver MR findings. Clin Imaging 1996; 20: 273-5.

17. Ferrozzi F, Bova D, Zangrandi A, et al. Primary liver leiomyosarcoma: CT appearance. Abdom Imaging 1996; 21: 157-60.

18. Sato S, Hosoi K, Kagawa T. A primary leiomyosarcoma of the liver: an autopsy report. Electron J Pathol Histol 2000; 6: 8.

19. Tsuji M, Takenaka R, Kashihara T, et al. Primary hepatic leiomyosarcoma in a patient with hepatitis C virus-related liver cirrhosis. Pathol Int 2000; 50: 41-7.

20. Iordanidis F, Hytiroglou P, Drevelegas A, et al. A 25-year-old man with a large hepatic tumor and multiple nodular lesions. Semin Liver Dis 2002; 22: 97-102.

21. Fujita $H$, Kiriyama $M$, Kawamura $T$, et al. Primary hepatic leiomyosarcoma in a woman after renal transplantation: report of a case. Surg Today 2002; 32: 446-9.

22. Lee HJ, Lee JL, Choi WH. A case of primary myxoid leiomyosarcoma of the liver. Korean J Intern Med 2002; 17: 278-82.

23. Almogy G, Lieberman S, Gips $M$, et al. Clinical outcomes of surgical resections for primary liver sarcoma in adults: results from a single centre. Eur J Surg Oncol 2004; 30: 421-7.

24. Jeong TY, Kim YS, Park KJ, et al. A case of primary leiomyosarcoma of the liver presenting with acute bleeding. Korean J Gastroenterol 2008; 51: 194-8.

25. Matthaei $H$, Krieg A, Schmelzle $M$, et al. Long-term survival after surgery for primary hepatic sarcoma in adults. Arch Surg 2009; 144: 339-44.

26. Giuliante F, Sarno G, Ardito F, et al. Primary hepatic leiomyosarcoma in a young man after Hodgkin's disease: diagnostic pitfalls and therapeutic challenge. Tumori 2009; 95: 374-7.

27. Liang X, Xiao-Min S, Jiang-Ping X, et al. Liver transplantation for primary hepatic leiomyosarcoma: a case report and review of the literatures. Med Oncol 2010; 27: 1269-72.

28. Shamseddine A, Faraj W, Mukherji D, et al. Unusually young age distribution of primary hepatic leiomyosarcoma: case series and review of the adult literature. World J Surg Oncol 2010; 8: 56.

29. Shivathirthan N, Kita J, Iso Y, et al. Primary hepatic leiomyosarcoma: Case report and literature review. World J Gastrointest Oncol 2011; 3: 148-52.

30. Takehara K, Aoki H, Takehara Y, et al. Primary hepatic leiomyosarcoma with liver metastasis of rectal cancer. World J Gastroenterol 2012; 18: 5479-84.

31. Tsai PS, Yeh TC, Shih SL. Primary hepatic leiomyosarcoma in a 5-monthold female infant. Acta Radiol Short Rep. 2013;2:2047981613498722. 
32. Chelimilla $H$, Badipatla $K$, Ihimoyan A, et al. A rare occurrence of primary hepatic leiomyosarcoma associated with epstein barr virus infection in an AIDS patient. Case Rep Gastrointest Med 2013; 2013: 691862.

33. Metta $\mathrm{H}$, Corti $\mathrm{M}$, Trione $\mathrm{N}$, et al. Primary hepatic leiomyosarcomaa rare neoplasm in an adult patient with AIDS: second case report and literature review. J Gastrointest Cancer 2014; 45: 36-9.

34. Majumder S, Dedania B, Rezaizadeh $\mathrm{H}$, et al. Tumor rupture as the initial manifestation of primary hepatic leiomyosarcoma. Gastrointest Cancer Res 2014; 7: 33-4.

35. Lin YH, Lin CC, Concejero AM, et al. Surgical experience of adult primary hepatic sarcomas. World J Surg Oncol 2015; 13: 87.

36. Hamed MO, Roberts KJ, Merchant W, et al. Contemporary management and classification of hepatic leiomyosarcoma. HPB (Oxford) 2015; 17: 362-7.
37. Lv WF, Han JK, Cheng DL, et al. Imaging features of primary hepatic leiomyosarcoma: A case report and review of literature. Oncol Lett 2015; 9 : 2256-60.

38. López-López V, Robles R, Ferri B, et al. Laparoscopically assisted resection of a primary hepatic leiomyosarcoma: A safe approach in a rare malignancy. Cir Esp 2017; 95(8): 478-80.

39. Xie $P$, Zhuang $H$. FDG PET/CT findings of primary hepatic leiomyosarcoma in an immunocompetent pediatric patient. Clin Nucl Med 2017; 42 323-4.

40. lida T, Maeda T, Amari Y, et al. Primary hepatic leiomyosarcoma in a patient with autosomal dominant polycystic kidney disease. CEN Case Rep 2017; 6: 74-8. 